\title{
Performance analysis of multipath ATM switches under correlated and uncorrelated IBP traffic patterns
}

\author{
A.-L. Beylot \\ Laboratoire PRiSM, Université de Versailles \\ 45 Avenue des Etats-Unis, 78035 Versailles Cedex - FRANCE \\ e-mail : beylot@prism.uvsq.fr, Tel (+33) 139254059 \\ M. Becker \\ Institut National des Télécommunications, \\ 9 rue Charles Fourier, 91011 Evry Cedex - FRANCE \\ and Associate member of MASI Lab. \\ 5 place Jussieu, 75230 Paris Cedex, FRANCE \\ e-mail : mbecker@int-evry.fr, Tel : (+33) 160764781
}

\begin{abstract}
An ATM Clos switch under bursty Interrupted Bernoulli Processes is studied at the cell level. Different correlated and uncorrelated traffic patterns are considered : cells of a given burst may or may not belong to the same VP/VC and be directed to the same output port of the switch. Uniform traffic, SSSD (Single Source to Single Destination) and a SSSD high traffic embedded in a uniform traffic are considered. Cells of a given burst are routed independently. So a resequencing mechanism has to be implemented. Approximate analytical models of the switch are proposed, they are validated by discrete event simulations for the parameter values for which simulations can be run. It is shown that such interconnection networks lead to good performance results even with small buffers under those different traffic patterns.
\end{abstract}

Key-words

A.T.M, switches, analytic models, bursty traffic, finite capacity queue.

Performance of Information and Communication Systems U. Körner \& A. Nilsson (Eds.) CC 1998 IFIP. Published by Chapman \& Hall 


\section{INTRODUCTION}

Performance of ATM networks will depend on transmission and switching performance. Many ATM switch designs have been proposed. There are monopath networks (Banyan, Delta, Omega networks) and multi-path networks (Clos, Benes networks). The first ones are easy to design ; a self routing algorithm can be used to route cells in the switch. For multipath networks, several complex algorithms may be implemented (cell or call based). The main problem when dimensionning ATM networks is due to the fact that traffic is not well characterized and that input traffics into units of the network are mostly superposition of output traffics from other units.

Several models have been proposed for interconnection networks, especially for Banyan Networks. But, in most of these papers, the authors studied the influence of the burstiness of sources but did not take into account the correlation between the destinations chosen by consecutive cells of a given burst. Input traffic was modelled by ON/OFF Markov processes (Bassi 1992) (De Marco 1994) (Morris 1992) (Theimer 1994). The interstage traffic was modelled by a Markov chain with several states which captures the burstiness of this traffic. The parameters of those Markov chains are fitted to the actual output traffic (first moments of the distribution of the busy period, of the idle period and of the time between two consecutive cells). This method is valid if it is possible to characterize the input traffic into one output queue of the following stage. This is wrong when considering that cells belonging to the same burst are directed to the same output since consecutive cells will be correlated. In those models, the burstiness of sources will be mostly absorbed by the splitting effect. Consequently, results are not far from results obtained using Bernoulli input traffics and Bernoulli interstage approximations.

Studies concerning the dissymetry of the traffic were performed for bufferless switches especially for Single Source to Single Destination (SSSD) traffics or Hot Spot traffics (Kim 1988) (Kim 1991). It was shown that mono-path networks lead to bad performance results because it is difficult to find a path for each incoming cell. The examples of non-uniform traffic patterns can be classified as follows :

- Non-uniform destination distribution

- SSSD type : each input port sends most or all of its cells to one output port (Chen 1992), (Kim 1988),

- Hot-Spot Traffic pattern : one (or several) output has a higher access load (Chen 1992), (Bassi 1992).

- Non-uniform input process : in (Kim 1988), one input port sends its cells to one output port and receives a heavier load. In (Morris 1992) input processes are IBP type; they differ by the squared coefficient of variation.

In this paper, we consider bursty input traffics (IBP type). Three traffic patterns are studied. The first one is the "classical" uniform traffic pattern case. 
In the second one, cells of a given burst will be directed to the same output port. In this SSSD traffic case, at a given time $t$, an output port of the switch is chosen by, at most, one burst. Consequently, "new" bursts are directed to "idle" ports of the switch (i.e. no burst is directed to this output port). This traffic case has been studied in a previous work (Beylot 1996) with a monopath interconnection network. It was shown that such networks lead to bad performance results : as soon as two bursts compeat for a common link inside the switch, the corresponding queue increases and cells are lost.

In the last traffic pattern, a case of non-uniform destination distribution and nonuniform input process has been studied. One hot-spot output port is considered, it corresponds to one high load input port. It is an SSSD high traffic embedded in uniform low traffic.

In the present work, we consider a switch based on a multipath interconnection network. Cells of a given burst are assumed to be routed independently. Consequently, it is necessary to reorder cells of a given burst. The resequencing cost is estimated by discrete event simulations. Approximate analytical models are proposed for the switch itself for the different traffic cases to evaluate the cell delay and the cell loss probability. They are validated by simulations.

The paper is organized as follows. Section 2 will present the interconnection network and the different traffic cases. In section 3 analytical models in the different traffic conditions are described. Results for the whole switch are presented in section 4. Approximations used in the analytical method will be discussed. From this study a dimensionning of the multistage network might be derived. It will be possible to answer the questions: "Does this multistage switch have good performance? Is resequencing bad for the performance?". Finally, section 5 summarizes our results and outlines directions for future works.

\section{SWITCH ARCHITECTURE AND OPERATION}

Let us consider a switch based on a three-stage Clos network (Clos 1953). The global number of input/output ports of the switch is $N$, the number of input ports of the first stage switching elements is $a$ and the number of paths $b(C(N, a, b)$ configuration). Let us sum up the main characteristics of the considered switch :

- Switching elements of a given stage are identical

- They include dedicated output FIFO queues with finite capacity

- No backpressure signals are exchanged between adjacent stages

- In each stage departure are taken into account before arrivals

- Internal, input and output links are assumed to have the same throughput.

In the first traffic case, let us assume that each input link is offered the same traffic load, destination addresses of the cells are uniformly distributed over all the output links of the network. Clos networks are multi-path. We choose the random policy in the present work : the choice of the matrix of the second stage is uniformly (and randomly) done. A reordering mechanism should be implemented. 
But, since the number of input/ouput ports of the switch is large, this cost is negligible (Kohlenberg 1996). It is not evaluated in this paper.

In the second traffic case, let us assume again that each input link is offered the same traffic load, cells of a given burst are assumed to be directed to the same output port of the switch. In this case a random routing policy has also been chosen. An algorithm based on burst routing may lead to well known results on non-blocking Clos switches, as far as the number of paths in the switch $b$ is greater or equal to $2 a-1$, the cell loss probability is 0 and the cell delay equal to 3 time slots. This is too optimistic because the input traffic will not be exactly SSSD.

A resequencing mechanism has to be implemented to reorder cells of a given connection. This mechanism may be quite complicated. In the present work, since only one burst may be directed to a given output port, cells of this given burst may be numbered when they enter the switch. In the resequencing buffer, cells may be ordered according to this number. The resequencing queue has two parameters : its capacity $C_{w}$ and its timeout $T_{w}$. Losses in the switch increase significantly the number of cells in the resequencer, since the resequencer will wait for lost cells and keep the following cells in order to resequence them afterwards. The present algorithm is not of general purpose. In a real switch implementation, a common buffer should be implemented and several bursts should be managed.

In the last traffic case, a SSSD IBP traffic (high traffic) is embedded in a uniform IBP traffic (low traffic). In our simulations, resequencing will be operated only for high traffic. The low load and the uniform distribution implies that low traffic generally, does not need to be resequenced. The cost is negligible. The previous algorithm has been adapted. The only difference corresponds to the low traffic cells directed to the hot spot output port. In the output queue designed for high traffic, if a cell of low traffic arrives, it will be transmitted on the output link.

ANALYTICAL MODEL OF THE SWITCH

\subsection{Traffic hypotheses and characterization}

IBP processes are discrete time "ON/OFF" processes. During the "ON" period, a packet is emitted according to a Bernoulli process (parameter $\alpha$ ). "ON" and "OFF" periods are geometrically distributed with parameter $p$ and $q$. The rate $\lambda$, the mean burst length $L_{B}$ and of the mean silence length $L_{S}$ of IBP sources are :

$$
\lambda=\alpha \operatorname{Pr}[" O N "]=\alpha \frac{1-q}{2-p-q} L_{B}=\frac{1}{1-p} L_{s}=\frac{1}{1-q}
$$

\subsection{Model of the uniform traffic case}

Let us first consider a first stage switching element. As the choice of the output is random and equidistributed, the splitted process produced by an IBP process on input link $m$ is an IBP process with parameters $(p, q, \alpha / b)$ where $b$ is the number 
of output ports of the switching element. The output queue is then an $n-\mathrm{IBP} / \mathrm{D} / 1 / M$ queue with departures before arrivals. The analysis of this queue has been presented in (Beylot 1998). The output traffic of such a queue is a D-MAP (Discrete Markov Arrival) process (Blondia 1992) but the number of states of this process is too large (equal to the number of states of the preceding Markov chain). Let us approximate the output process of such queues by an IBP process (Beylot 1998). The parameters are fitted to the actual interdeparture time of cells. From this approximation of the output process of the first stage switching element, a solution of the second stage will be derived. The solution is then iterated for the third stage. It leads to a model of the whole switch.

\subsection{Model of the switch in the SSSD Traffic case}

Cells within bursts are randomly directed over the output queues of the first stage switching elements. Consequently, an output queue of a first stage switching element can again be modelled by an $n-\mathrm{IBP} / \mathrm{D} / 1 / M$ queue.

The output traffic of a first stage switching element will be splitted and the input traffic into an output queue of the second stage cannot be derived from the previous study (it should be necessary to know to which output port, cells are directed, the choice is not random anymore). In fact, let us focus on an output queue of the second stage. It receives cells coming from all the input ports of the switch directed to a given switching element of the third stage that have chosen the output port of the first stage connected to the tagged switching element. In the SSSD traffic case, it receives at most cells belonging to $a$ bursts directed to the corresponding output ports of the third stage. The input traffic offered to a given output port of a second stage switching element can consequently be modelled by the superposition of $a$ IBP processes with parameters $p, q, \alpha / b$ because of the splitting effect of the first stage. Those input processes will be modified by the first stage queue. So let us consider an output traffic of the first stage switching element. It corresponds to the superposition of $a$ input traffics. As in 3.2, it can be approximated by an IBP traffic with parameters $p_{\text {ou }}, q_{\text {ou }}, \alpha_{\text {ou }}$ and consequently an input process modified by a first stage switching element can be modelled by an IBP process with parameters $p_{\text {out }}, q_{\text {out }}, \alpha_{\text {out }} / a$.

An approximate model of the second stage will consequently be derived from the study of an $a-\mathrm{IBP} / \mathrm{D} / 1 / M$ with such parameters. Let us note that in this approximate model we did not take into account the fact that several bursts from a given switching element of the first stage may be directed to the same output port of the second stage.

When considering an output queue of the third stage, it appears that it receives cells from one input port of the switch. Let us model the first two stages by a queue with $b$ servers, each server corresponds to a path. The mean response time of this queue corresponds to the mean response time derived from the analytical models of the first two stages. This response time is mainly composed of two parts : the 
service time equal to 2 time slots and the waiting time experienced in the first two stage queues. Let us approximate this response time by a Geo+D process. The deterministic part of this response time corresponds to the service time and the geometrical part to the waiting time. The third stage is modelled by a constant service time and finite capacity queue. Figure 1 shows the model used to study the performance of the third stage. The source is an IBP / Geo $+\mathrm{D} / b / b$ queue. It will be valid as long as the cell loss probability of the first two stages is negligible. Details of this study are presented in (Beylot 1998).

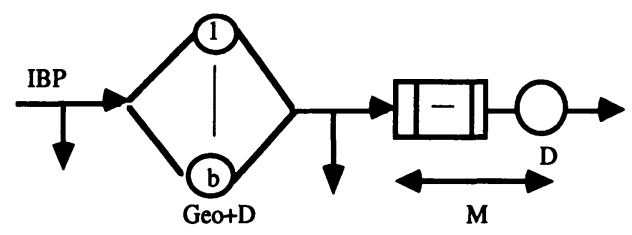

Figure 1 Model of the third stage.

\subsection{Model of the SSSD + uniform traffic case}

In this traffic case, using IBP approximations for the interstage traffics, analysis of the different stages can be done by the study of $n-\mathrm{IBP} / \mathrm{D} / 1 / M$ and $\mathrm{IBP}_{1}+n-\mathrm{IBP}_{2} / \mathrm{D} / 1 / M$ queues. Details about different traffic types on different queues and the abalysis of the $\mathrm{IBP}_{1}+n-\mathrm{IBP}_{2} / \mathrm{D} / 1 / M$ queue are presented in (Beylot 1998). On the last stage splitted processes corresponding to the same burst (high traffic) will go to the same output, so it would be very wrong to assume them to be independent. The solution that is proposed here is to consider that at one time there will be only one input link on which the burst will arrive.

For each low traffic cell the choice of the output by each cell from one burst is random. Consequently, the independence assumption is not bad for the low traffic. So, we approximate input processes on the different links by assuming that on one link, there is a superposition of high traffic and of one low load process. It is a DBMAP process. The performance criteria on the last stage will be derived from the solution of this DBMAP $+n$ IBP/D/1/M queue.

\section{$4 \quad$ RESULTS}

Several parameter values are chosen. The $\mathrm{C}(128,4,8)$ configuration has been chosen (Beylot 1995). The whole cell loss probability on the three stages and the cell delay across the whole switch are represented for the approximate analytical model and the simulation as a function of the memory size of the first stage. The memory size on the first stage varies and the best value for the memory sizes of the second and of the third stage, for a given global memory size, is derived from the analytical model (the best value is the one that leads to the lowest loss probability). 
The points in the analytical model are validated by discrete event simulations. Confidence intervals are around $10-20 \%$. For cell loss probability of $10^{-7}$ there are $20 \%$ confidence interval. For cell loss probability higher than $10^{-6}$ they are around $10 \%$. Let us note $\mathrm{A}$ : the analytical model and $\mathrm{S}:$ the simulations.

\subsection{Results - Uniform Traffic Case}

The global memory size in the switch is $128 \times 72$. The input load is 0.8 .

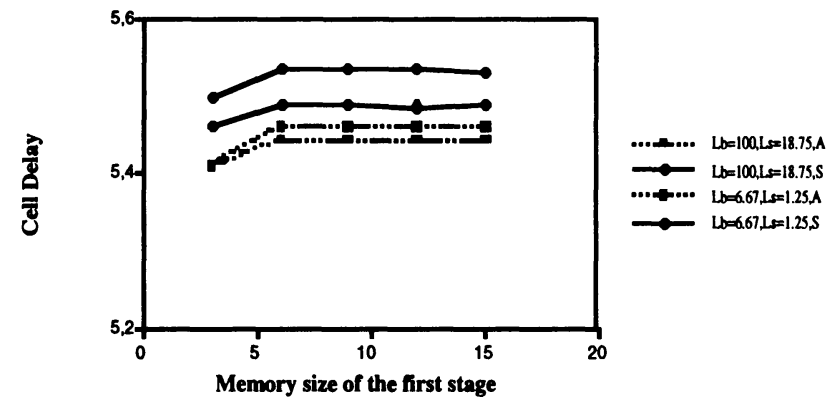

Figure 2 Cell delay as a function of the memory size of the first stage, $\alpha=0.95$ Uniform Traffic.

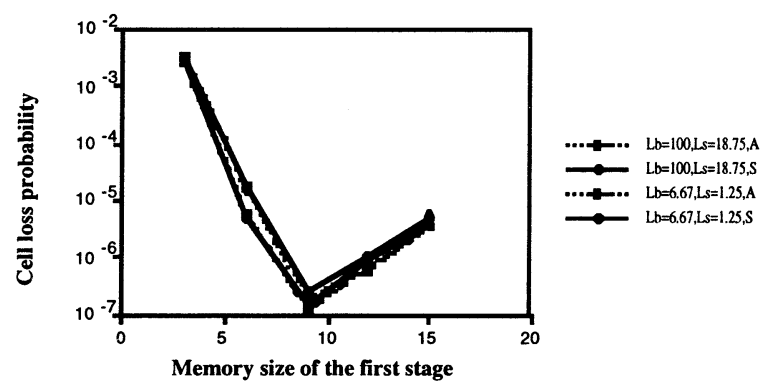

Figure 3 Cell loss Probability as a function of the memory size of the first stage, $\alpha=0.95$ - Uniform Traffic.

Figure 2 presents the cell delay and the cell loss probability as a function of the memory size of the first stage. The switch is heavily loaded (load is 0.8). Approximate analytical solution results and simulation results are given for two traffic cases : $L_{b}=100, L_{s}=18.7, \alpha=0.95$ and $L_{b}=6.67, L_{s}=1.25, \alpha=0.95$. For the same traffic parameters, the two simulation curves are nearly the same and the two analytical models are also very much the same. So it appears that the burstiness has not much influence on the delay (Figure 2). Each analytical curve is 
not far from the simulation curve (1\%), this validates for the chosen values of the parameters the approximate analytical model. It appears that the cell loss probability does not depend on the burstiness nor on the peak rate for a given load, but depends much on the memory repartition (Figure 3). The best memory repartition is 9-10-34. For the chosen values of the parameters, the simulation results are not far from the analytical model ; so it appears that the approximate analytical model is validated. This traffic case is not the most realistic one.

\subsection{Results - SSSD Traffic case}

In this traffic case, the global memory size inside the swich is $128 \times 36$. The mean input rate is 0.72 and the mean burst length is 100 . The peak rate is 0.9 . Figure 4 shows the cell delay and the cell loss probability within the switched. The cell delay do not depend on the memory repartition. Cell delay is around 4 time slots and is well approximated by our analytical model. The best memory configuration is (8-7-6). In this case the cell loss probability within the switch is approximately $10^{-6}$. The cell loss probability is well approximated by our analytical model.

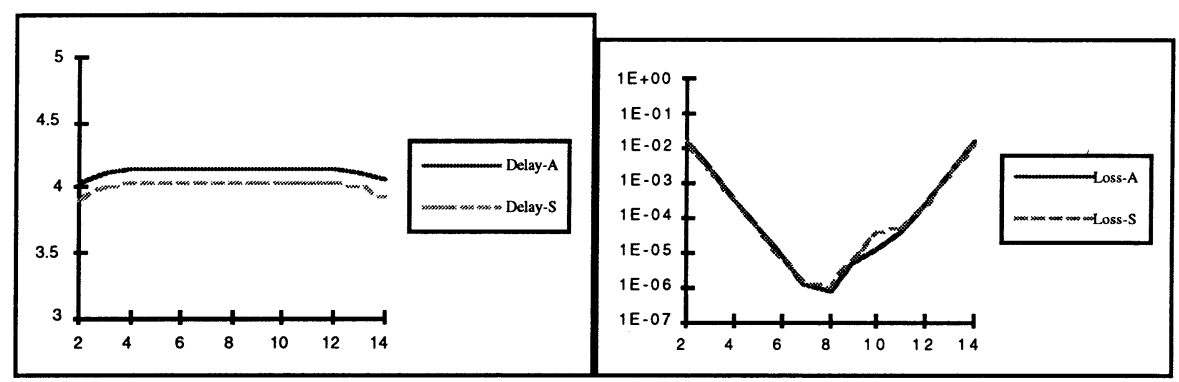

Figure 4 Cell Delay and Cell Loss Probability as a function of the memory size on the first stage, $L_{b}=100, L_{s}=25, \alpha=0.9$ - SSSD Traffic.

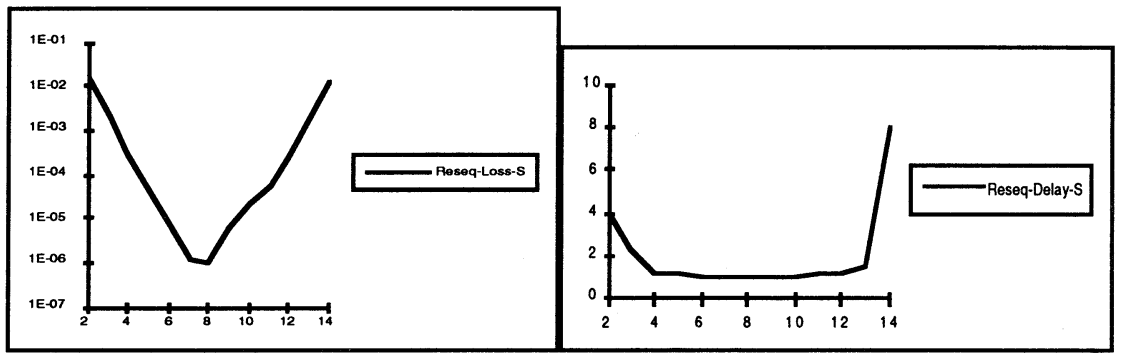

Figure 5 Resequencing Cell loss probability and Resequencing Cell Loss Probability as a function of the memory size on the first stage $-C_{w}=10, T_{w}=10$, $L_{b}=100, L_{s}=25, \alpha=0.9$ - SSSD Traffic. 
Let us analyze now the performance of the resequencing unit. Figure 5 shows the resequencing delay and the resequencing cell loss probability obtained by simulations. The resequencing buffer size $C_{w}$ is 10 . The value of the time-out $T_{w}$ is also equal to 10 . With those parameter values, the cell loss probability inside the switch and within the resequencer buffer are of the same order of magnitude. It is shown that when the cell loss probability within the switch is high, the mean resequencing time and the resequencing cell loss probability are quite high because the resequencing unit waits for lost cells.

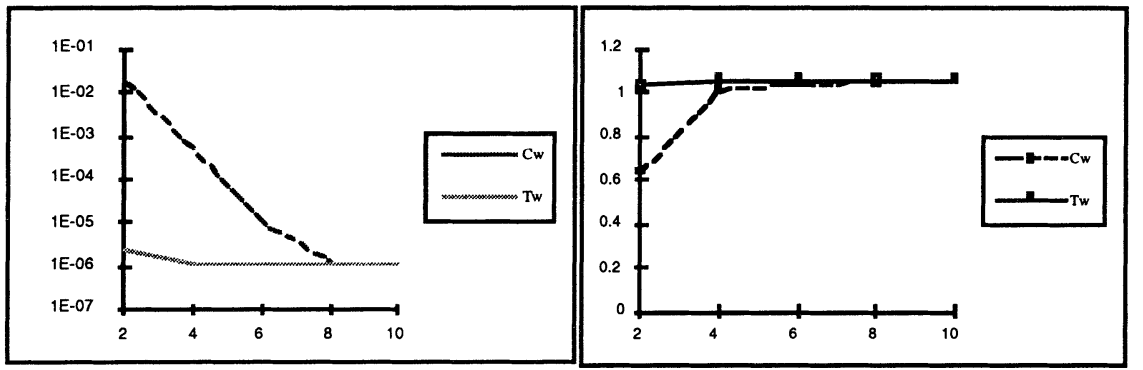

Figure 6 Resequencing Cell loss probability and Delay as a function of $C_{w}$ $\left(T_{w}=10\right)$ and of $T_{w}\left(C_{w}=10\right), L_{b}=100, L_{s}=25, \alpha=0.9$ - SSSD Traffic.

Let us analyze now the influence of the parameters $C_{w}$ and $T_{w}$. We only investigated the case when the memory in the different stages is (8-7-6) i.e. the case when the cell loss probability within the switch is quite low. It is shown (Figure 6) that the performance of the resequencer mainly depends on the resequencer buffer size. The cell loss probability decreases with $C_{w}$ (in this case $T_{w}=10$ ). The analysis of the influence of the time out parameter is quite different. In the case when $C_{w}=10$, the cell loss probability is higher when $T_{w}=2$. It seems that when $T_{w}=4$, the best performance of the resequencing algorithm is reached. It remains constant and does not improve when $T_{w}$ is larger than 4 .

\subsection{Results - SSSD Traffic embedded in a uniform traffic}

The global memory size in the switch is $128 \times 48$. Let us note High(Low)- $\mathrm{A}=$ the analytical results for high(low) traffic; High(Low)-S = the simulation results. Let index $h$ (resp. $l$ ) respectively refer to high (resp. low) traffic. An output port will be heavily loaded, so it is necessary to choose parameters such than the load is not more than 1 on this output link. The following parameters are considered in Figures 7 and 8 (the output traffic rate will be quite high on the hot-spot):

- low traffic: $\lambda_{l}=0.1, p_{l}=0.99, q_{l}=0.99875, \alpha_{l}=0.9$

- high traffic: $\lambda_{h}=0.54, p_{h}=0.99, q_{h}=0.985, \alpha_{h}=0.9$ 

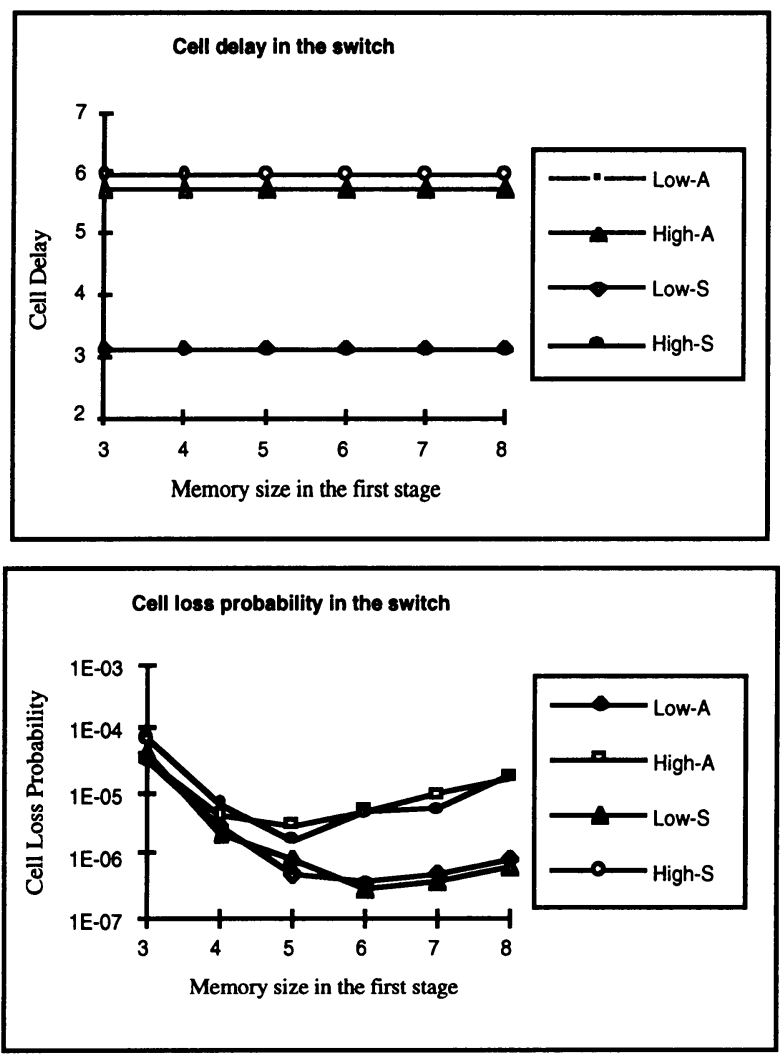

Figure 7 Cell delay and Cell Loss Probability in the switch, $\lambda_{h}=0.54, \lambda_{l}=0.1$; $L_{b, h}=L_{b, l}=100$ - Low load SSSD Traffic embedded in a uniform traffic.

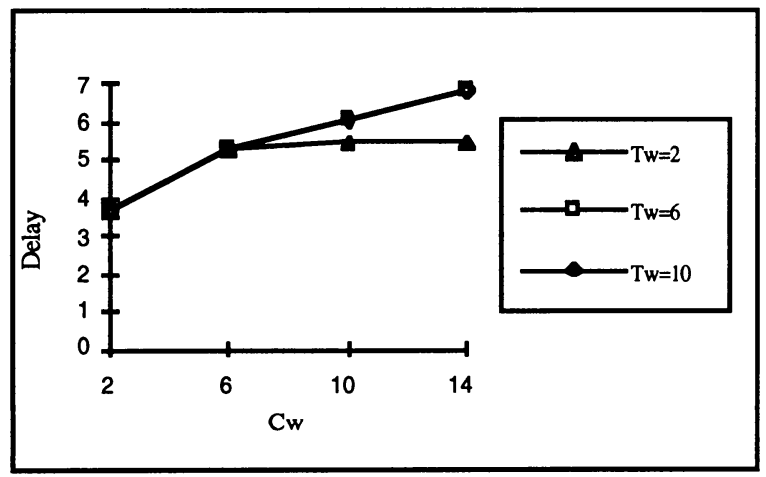

Figure 8 Cell Delay in Resequencer for high traffic - Low load mixed traffic. 
Let us note that an estimation of the number of desequenced cells is $2 \%$. Using the same burst length with traffic rates equal to 0.54 and 0.36 , it is around 7 or $8 \%$. So resequencing does not cost much and since it was shown that this switch is performant it appears to be good to choose this multipath switch.

\section{CONCLUSION}

A multipath ATM switch performance has been studied under non-uniform traffic patterns. These hypotheses for destinations are reasonable, because of reservations in VPs and VCs of ATM networks. Uniform traffic destination has first been considered to extend the known results to multipath switches. It was shown that the burstiness of the sources is mostly absorbed by the splitting effect. This traffic case is too optimistic and simple Bernoulli approximations would nearly lead to the same results.

Single Source to Single Destination (SSSD) and SSSD embedded in a uniform traffic were then considered. Analytical model were derived for the different traffic cases. The approximation of most of the interstage traffics by IBP fitting traffic seems to be working. The proposed solutions gave good performance results. The results show that buffer placement in the switch is very important. This stands at least for the parameter values for which simulations may be performed. Traffic assumptions are realistic in this case. Performance results are good in the SSSD case. For SSSD imbedded into a uniform traffic, performance is good as long as for the heavily loaded output link the load is not prohibitive.

The Clos switch is multipath. An independent routing for each cell of the same burst has been assumed. It is necessary to reorder incoming cells. A study of some performance results of a simple resequencing algorithm has been estimated. Our aim was not do design a resequencing algorithm ; it was to estimate the order of magnitude of the resequencing cost, in these traffic conditions. In any case, the performance is not bad, even with small resequencing buffers. It might be interesting to solve the resequencer model.

As a conclusion it appears that this multipath switch and the cell routing inside the switch avoid the congestion problems that appears in a monopath switch (Beylot 1996). A Clos network seems to be a proper choice for a switch.

\section{REFERENCES}

Bassi S., Decina M., Pattavina A. (1992) Performance analysis of the ATM shuffleout switching architecture under non-uniform traffic patterns, in Proceedings of IEEE Infocom'92.

Beylot A.-L., Becker M. (1995) Dimensioning an ATM switch based on a threestage Clos interconnection network, Annals of Telecom., 50(7-8), 652-666. 
Beylot A.-L., Harfouche I., Becker M. (1996) Performance Analysis of monopath ATM switches under correlated and uncorrelated traffic patterns, in 5th Open Workshop on High Speed Networks ENST/Stuttgart Univ., Paris.

Beylot A.-L., Becker M. (1998) Performance analysis of multipath ATM switches under correlated and uncorrelated IBP traffic patterns with independent routing and with resequencing, PRiSM research report 98-04.

Blondia C., Casals O. (1992) Statistical Multiplexing of VBR sources: A matrixanalytic Approach", Performance Evaluation, 16(1-3), 5-20.

Chen D., Mark J. (1992) A buffer management scheme for the SCOQ switch under non-uniform traffic loading", in Proceedings of IEEE Infocom'92, paper 1D.4.

Clos C. (1953) A study of non-blocking Switching Networks", Bell System Tech. 32, 406-424.

De Marco M., Pattavina A. (1994) Performance Analysis of ATM multistage networks with shared queueing under correlated traffic, in Proceedings of ITC'14, 601-610, Juan les Pins.

Kim H.S., Leon-Garcia A. (1988) Performance of Buffered Banyan Networks under Non Uniform Traffic Patterns, in Proceedings of Infocom 88, 344-353

Kim H.S., Widjaja M.G., Leon-Garcia A. (1991) Performance analysis of output buffered Banyan Networks with arbitrary buffer sizes, in Proc. of Infocom' 91.

Kolhenberg I. (1996) Performances des commutateurs ATM dans des conditions non-uniformes de fonctionnement, Phd Report Thesis, University of Paris 6.

Morris T., Perros H. (1992) Performance Modelling of a multi-buffered Banyan Switch under Bursty Traffic, in Proceedings of IEEE Infocom'92, paper 3D.2.

Theimer T. (1994) A New Model for the Analysis of Multistage ATM Fabrics, in Proceedings of ITC'14, Juan Les Pins.

\section{BIOGRAPHY}

André-Luc Beylot received the engineer degree from the Institut d'Informatique d'Entreprise in 1989 and the Ph.D. degree in computer science from the University of Paris VI in 1993. From 1993 to 1995, he worked at the Institut National des Telecommunications, and from 1995 to 1996 at CNET (France Telecom Research Laboratory) in Rennes. Since 1996, he is an associate professor at the University of Versailles. His interests are in the performance evaluation of communication networks, especially with regard to ATM networks.

Monique Becker graduated from Ecole Normale Supérieure de Jeunes Filles in 1968, passed the mathematics "agrégation" and received the State Doctorate degree from the University of Paris VI in 1976. She joined the National Center of Scientific Research where she had the responsability for a group of researchers working on performance evaluation. In 1987, she joined France Telecom University where she got the position of Professor and Chairman of the Computer Science Department. She is managing a group of researchers of the Department (including professors and Phd students) working on performance evaluation of computer networks. Their main interest concerns ATM networks. 